doi: https://doi.org/10.31434/rms.v4i3.182 correo : revistamedicasinergia@gmail.com

\title{
Esclerosis tuberosa
}

\author{
Tuberous sclerosis
}

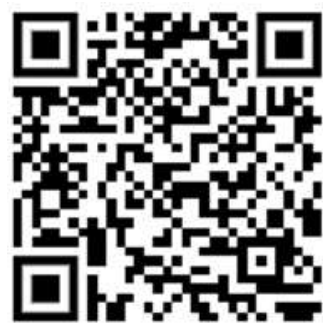

RECIBIDO

$24 / 1 / 2019$

\author{
${ }^{1}$ Dr. Marco Tulio Gómez Cerdas \\ Clínica Carlos Durán Cartin, San José, Costa Rica \\ mac11@hotmail.com \\ https://orcid.org/0000-0002-5329-3359
}

${ }^{2}$ Dr. Johnny Alejandro Vargas Morales Clínica Carlos Durán Cartin, San José, Costa Rica alejhovarmor@hotmail.com

https://orcid.org/0000-0002-9521-5041

CORREGIDO

ACEPTADO

9/2/2019

$20 / 2 / 2019$

\section{RESUMEN}

La esclerosis tuberosa es un trastorno multisistémico que presenta un patrón de herencia autosómico dominante y se caracteriza por la presencia de crecimiento de hamartomas en cerebro, ojos, piel, riñones, corazón y pulmón. Se debe a la mutación heterocigótica en uno de los dos genes supresores de tumor TSC1 y TSC2. Las manifestaciones clínicas varían entre los pacientes, por lo que se han consensuado criterios clínicos y radiológicos para establecer su diagnóstico. Se requiere de un equipo multidisciplinario familiarizado en la evaluación clínica, para establecer un tratamiento adecuado y así optimizar y garantizar una mejor calidad de vida en estos pacientes.

PALABRAS CLAVES: síndrome; esclerosis tuberosa; manifestaciones neurológicas; epilepsia.

\section{ABSTRACT}

${ }^{1}$ Médico general, graduado de la Universidad de Iberoamérica (UNIBE), médico en Clínica Carlos Durán Cartin, San José, Costa Rica. Código médico:15082 mgc11@hotmail.com

${ }^{2}$ Médico general, graduado de la Universidad de Ciencias Médicas (UCIMED), médico en Clínica Carlos Durán Cartin, San José, Costa Rica. Código médico:15058 alejhovarmor@hotmail.com

Tuberous sclerosis is a multisystem disorder that presents an autosomal dominant pattern of inheritance and is characterized by the presence of hamartomas in brain, eyes, skin, kidneys, heart and lungs. It is due to a heterozygous mutation in one of the two tumor suppressor genes TSC1 and TSC2. The clinical manifestations vary between patients, and therefore, there exist consensus diagnostic clinical and radiological criteria for diagnosis. It requires a multidisciplinary team familiar with the clinical evaluation, to establish an adequate treatment and thus optimize and guarantee a better quality of life in these patients.

KEYWORDS: syndrome; tuberous sclerosis, neurologic manifestations; epilepsia 


\section{INTRODUCCIÓN}

La esclerosis tuberosa (ET) 0 el complejo de esclerosis tuberosa (CET), fue descrita por Bourneville en 1880; él describió en estudios post mortem, con pacientes que sufrían de convulsiones y retraso mental, logrando identificar características patológicas típicas de la enfermedad, por ejemplo: tumores amarillentos en los riñones, áreas escleróticas en el cerebro y múltiples tumores blanquecinos en los ventrículos laterales, razón por la cual Bourneville acuñó el término esclerosis tuberosa de las circunvoluciones cerebrales $(1,2,3)$.

Posteriormente, en 1908, Heinrich Vogt describió la tríada clásica o tríada de Vogt, constituida por epilepsia, discapacidad intelectual y angiofibromas. En 1921, Van der Hoeve fue el primero en describir los hamartomas en retina y también se le acredita acuñar el término facomatosis (del griego:phako lentes, oma tumor, y osis condición) para síndromes neuroóculo-cutáneos. La asociación de las máculas hipocrómicas (en hoja de fresno) presentes en más de $90 \%$ de los afectados, no fue reconocida hasta 1932, por Critchley y Earl. Luego, Gold y Freeman junto con Fitzpatrick y colaboradores establecieron que estas lesiones hipocrómicas son un signo temprano e importante $(4,5)$.

El nombre Esclerosis Tuberosa se compone la palabra latina tuber (crecimiento en forma de raíz) y la palabra griega skleros (duro), para referirse a las lesiones gruesas y firmes llamadas túberes (4).

A esta patología se le considera como un síndrome neurocutáneo multisistémico, producido por un padecimiento genético hereditario de carácter autosómico dominante de alta penetrancia y una expresividad variable, afecta a niños y adultos, afectación a muchos órganos, por ejemplo, tumores (hamartomas) en la piel, riñones, cerebro, corazón, ojos, pulmones o cavidad oral; puede incluir trastornos neurológicos incapacitantes, como epilepsia, retraso mental y autismo $(6,7,8)$.

En ocasiones el diagnóstico de esta enfermedad puede ser muy difícil debido a las manifestaciones clínicas tan distintas inclusive entre individuos con la misma mutación, por esto, en el presente artículo se exponen las principales características etiopatogénicas, criterios diagnósticos y posibles tratamientos terapéuticos (9).

\section{EPIDEMIOLOGÍA}

Es la segunda en frecuencia del grupo de las facomatosis 0 síndromes neurocutáneos, superada únicamente por la neurofibromatosis; es una enfermedad poco frecuente y se estima una prevalencia de 1 en 9000 individuos en la población en general y se ha estimado que su incidencia oscila entre 1 en 5000 a 10000 nacidos vivos $(1,8,10)$.

Se sabe que afecta por igual a todos los grupos étnicos y que su distribución es similar en ambos sexos (3).

En Inglaterra, Callaghan et al comunicaron una prevalencia de 4-5 por cada 100.000 habitantes. En otros estudios realizados en Polonia y Estados Unidos, se han registrado prevalencias de un caso por cada 6.000-10.000 persona (3).

En Latino América no existen muchos datos sobre la prevalencia, no obstante, se han documentado escasos reportes individuales, tal es el caso de 
Brasil, donde se registra una incidencia de 1 por 10 mil a 50 mil nacidos vivos, con mutaciones espontáneas en el $85 \%$ de los casos $(3,11)$.

En Costa Rica, se realizó un estudio con base a la población del Hospital de Nacional de Niños (HNN) con esta enfermedad, realizado durante el período 2000-2010, y se estimó una prevalencia de ET de 3,09 pacientes por cada 100.000 nacidos vivos. Dentro los criterios por los cuales estos pacientes fueron referidos para su estudio en neurología del HNN fueron: convulsiones (75\%), lesiones en la piel (11\%), rabdomiomas cardíacos (11\%), y macrosomía y vómitos (3\%) (3).

\section{ETIOPATOGENIA}

Es una enfermedad genética hereditaria, autosómica dominante; los genes involucrados son el TSC1 en el locus génico 9q34 (que codifica hamartina) o TSC2 (codificación de tuberina) el locus génico 16p13. En raros casos con deleciones de TSC2 abarcan el gen PKD2 presentando una enfermedad poliquística de inicio temprano grave $(1,3,6-8,12,13)$.

Los análisis genéticos han mostrado que estas mutaciones producen alteración en la migración y diferenciación de las células de la cresta neural, que a su vez tienen un origen ectodérmico y migran a múltiples órganos de diferente origen embriológico, llevando al desarrollo de tumores benignos, circunscritos, no invasivos en órganos de diferentes sistemas (1,3,6-8).

La hamartina y la tuberina interactúan al formar un complejo funcional denominado hamartina-tuberina y sus alteraciones interrumpen el proceso de señalización de la vía mTOR (por sus siglas en inglés, Mammalian Target of
Rapamycin), que es una serina/treonina cinasa, que tiene funciones pleiotrópicas debido a la participación en la regulación del inicio de la transcripción del ARNm y en la transducción de múltiples proteínas que intervienen en la organización del citoesqueleto, en el transporte a través de la membrana, en la degradación de proteínas, en la biogénesis de los ribosomas y en el control del ciclo celular (8).

El complejo hamartina-tuberina también efectúa la inhibición de la PI3K (fosfatidil inositol-3-quinasa) y en la activación de señales dependientes de la vía de insulina, que afectan a mTOR, y a las cascadas de señalización de otras quinasas, lo que modifica el crecimiento celular y la proliferación. Además, activa la GTPasa de Rheb (Ras homolog enriched in brain), que a su vez regula a mTOR; al perderse este control, se generan niveles anormalmente altos de mTOR y, por ende, la activación cascada abajo de la señalización. La tuberina también se une a la p27, que está implicada en la regulación del ciclo celular. Debido a las funciones que desempeñan TSC1 y TSC2, estos genes son considerados supresores tumorales (8).

En los pacientes con mutaciones TSC2 sufren de síntomas más severos que aquellos con mutaciones en TSC1, presentando tubérculos corticales, angiomiolipomas renales, hamartomas de la retina, y angiofibromas faciales. Se observa mayor epilepsia. farmacorresistente. También se han reportado casos en la literatura que ante la evidencia de rabdomiomas cardiacos, hasta un $88 \%$ de los fetos pueden presentar diagnóstico ET, a su vez, aproximadamente en el $30 \%$ los fetos con ET tendrán rabdomiomas. Se debe tener en cuenta, que este tipo 
de lesiones cardiacas van a tener una tendencia a la involución espontánea en la vida extrauterina, pero que in útero pueden crecer a lo largo de la gestación y producir obstrucción de los tractos de entrada y salida del corazón y llevar a la muerte del feto $(1,12)$.

\section{MANIFESTACIONES CLÍNICAS}

Las manifestaciones clínicas del ET son heterogéneas y se desarrollan principalmente en la infancia, por lo general antes de los 10 años de vida, manifestándose habitualmente con epilepsia, autismo y falla cardíaca. Por el contrario, en el adulto se caracteriza por la presencia de falla renal, alteraciones pulmonares 0 dermatológicas. Al ser su evolución de carácter progresivo, su expectativa de vida en la mayoría de los casos es de 35 años (11).

Los principales hallazgos clínicos descritos en orden de frecuencia son: lesiones dérmicas con más del $90 \%$, lesiones cerebrales $90 \%$, anormalidades renales $70-90 \%$, hamartomas retinianos 50\%, y rabdomiomas en aproximadamente 40$60 \%$ de los pacientes.

En la actualidad la tríada clásica (retraso mental, epilepsia y adenoma sebáceo) se manifiesta en menos de un tercio de los pacientes y hasta en $6 \%$ de los casos no se presentan ninguna de dichas características (11).

A continuación, se describen los principales hallazgos clínicos ordenadas por aparatos y sistemas:

\section{MANIFESTACIONES DERMATOLÓGICAS}

1. Máculas hipocrómicas: son las más frecuentes, encontrándose en el $90 \%$ de los casos. Se presentan desde el nacimiento o en la infancia, pudiendo manifestarse en forma única o múltiple, su localización habitual es en tronco 0 en extremidades (11.14).

El número varía desde unas pocas hasta docenas, pero si existen en número de 3 ó más, son un criterio diagnóstico mayor de ET. Pueden adoptar cualquier forma, pero clásicamente se han descrito como de forma parecida a la hoja del fresno, es decir, con un extremo puntiagudo y el otro redondeado. Pueden medir desde pocos milímetros hasta $5 \mathrm{~cm}$. Para observarlas puede ser precisa la aplicación de luz de Wood (lámpara que emite luz ultravioleta en el rango de 320-400 nm y que acentúa los trastornos de pigmentación epidérmicos). Cuando afectan al cuero cabelludo pueden asociarse con poliosis (mechón de cabellos hipopigmentados). En ocasiones son zonas hipopigmentadas de muy pocos milímetros, llamadas manchas en confeti, que se consideran un criterio menor de la enfermedad $(9,11)$.

2. Angiofibromas faciales: ocupan el segundo lugar en frecuencia, ocurren en aproximadamente el $75 \%$ de los pacientes, su comienzo habitual es entre los 2 a 5 años de edad y por lo general se presentan de forma múltiple, son pápulas del mismo color que la piel o más rojizas, dependiendo de la proporción de tejido conectivo y vasos sanguíneos que contengan. Típicamente se distribuyen por la nariz, pliegues nasogenianos, mejillas y mentón. Por lo general 
confluyen entre sí y se tornan en apariencia carnosa $(9,11)$.

Antes se les conocía como adenoma sebaceum, nombre inadecuado ya que no guardan ninguna relación con las glándulas sebáceas. Aunque la gran mayoría de pacientes con ET tiene múltiples angiofibromas faciales, se han descrito casos con pocas lesiones. Debido a que pueden observarse uno 0 dos angiofibromas esporádicos aislados en la población general, entre los criterios diagnósticos actuales se considera un criterio mayor la presencia de al menos 3 lesiones. En la enfermedad de Birt-HoggDubbé y en la neoplasia endocrina múltiple tipo 1 (MEN1) también se ha descrito la presencia de múltiples angiofibromas faciales, aunque la aparición de las lesiones suele ser más tardía (9).

3. Placas chagrín ("shagreen") piel de zapa o parche lijoso: son tumores de tejido conectivo, también llamados colagenomas, del mismo color de la piel circundante o amarronados, rugosa y elevada, es considerada un criterio mayor, siendo descrita en $65 \%$ de los casos, se localizada habitualmente en la región lumbosacra, pero pueden aparecer en cualquier zona. Aparecen durante la infancia. Suelen ser asintomáticos, pero en ocasiones pueden provocar prurito 0 disestesia $(9,11)$.

4. Placa fibrosa cefálica: ocurre en un $25 \%$ de los pacientes. La localización clásica es la frente, por lo que clásicamente se ha denominado placa frontal. En el último consenso de criterios diagnósticos se modificó el nombre a placa fibrosa cefálica ya que puede localizarse en cualquier zona del área craneofacial (9).

5. Fibromas ungueales (Tumores de Koenen): se presentan como unos nódulos rojizos o sonrosados que aparecen alrededor o bajo las uñas sin traumatismo previo. Pueden presentarse a partir de los 5 años de edad y a lo largo de la toda la edad adulta (4).

\section{MANIFESTACIONES DENTALES}

Dentro de las manifestaciones dentales que se describen con mayor frecuencia son la hipoplasia del esmalte dentario en forma de agujeros, pozos o depresiones, distribuidos de forma aleatoria y los fibromas intraorales, que aparecen mayormente en adultos que en niños, pudiendo presentarse en mucosa labial, bucal, gingival, etc (11). $\mathrm{Si}$ presentan 3 o más agujeros $\mathrm{u}$ hoyuelos dentarios, se considera un criterio diagnóstico menor de la enfermedad. En algunas series hasta el $100 \%$ de los individuos afectos presentan los defectos en el esmalte. Están ausentes en los dientes deciduales según algunos autores. También es un criterio diagnóstico menor la presencia de 2 o más fibromas orales (9).

\section{MANIFESTACIONES OFTÁLMICAS}

Las lesiones retinianas son descritas en el $87 \%$ de los pacientes, siendo los hamartomas astrocíticos retinianos los más frecuentes con una incidencia que oscila entre $30-50 \%$ de los casos. La lesión típica con aspecto de "palomita de maíz" es fácilmente reconocible en la papila óptica, pero son más frecuentes las formas planas semitransparentes locacalizadas en la 
retina media y en el polo posterior $(9,11)$.

Por lo general son múltiples, no alteran la visión y son considerados como un marcador confiable para su diagnóstico sobre todo en pacientes jóvenes que carecen de otras características. No obstante, es importante considerar que se han descrito casos de crecimiento y malignización de los hamartomas con complicaciones oculares graves secundarias como desprendimiento de retina o glaucoma neovascular que requieran tratamiento oftalmológico. Se consideran un criterio mayor en caso de presentar 2 o más lesiones $(9,11)$.

Otra de las manifestaciones clínicas oftalmológicas son las manchas acrómicas retinianas, estas ocurren en el $40 \%$ de los pacientes y se consideran un criterio diagnóstico menor (9).

\section{MANIFESTACIONES A NIVEL DEL SISTEMA NERVIOSO CENTRAL}

La principal anormalidad observada consiste en la presencia de células germinales displásicas. Dichas células no pueden diferenciarse, migrar ni organizarse normalmente, provocando una serie de alteraciones estructurales, neurológicas y neuropsiquiátricas que dependiendo de su severidad aumentan el riesgo de mobi-mortalidad (11).

Más del $90 \%$ de pacientes afectos con ET presentará las lesiones típicas de SNC que consisten en nódulos subependimarios, túberes y líneas de migración radial en la sustancia blanca. Aproximadamente un $10-20 \%$ de los afectos presentará un crecimiento progresivo de un nódulo subependimario hasta un tamaño superior a $10 \mathrm{~mm}$, lesión que constituye un astrocitoma subependimario de células gigantes (9).

Las características de las lesiones típicas de la ET en sistema nervioso central son:

1. Túberes corticales: se trata de displasias del córtex cerebral que suelen ser múltiples y pueden localizarse en cualquier zona cortical o subcortical. Suelen mantenerse estables a lo largo de la vida del paciente, aunque pueden calcificarse o sufrir degeneración quística. Rara vez malignizan, se asocian a alteraciones en la sustancia blanca, epilepsia, alteraciones del comportamiento y deterioro cognitivo. Pueden ser únicos o múltiples y su sitio habitual de aparición es en los lóbulos frontales (90\%), seguido de los lóbulos parietales, occipitales y temporales $(9,11,15)$.

En un $25 \%$ de los casos pueden afectar también a cerebelo, siempre en pacientes que también tienen afectación supratentorial y suelen calcificar de forma más intensa y precoz que los cerebrales. Es muy raro que afecten al tronco cerebral o a la médula espinal (9).

También se ha observado que los pacientes que presentan mayor número de túberes sufren un mayor grado de discapacidad intelectual. Se ha sugerido que los trastornos del espectro autista pueden ser más frecuentes en pacientes con más carga de túberes en los lóbulos temporales o con su presencia en cerebelo. Muchos túberes se asocian con alteraciones de la sustancia blanca subyacente denominadas líneas de migración radial y que frecuentemente 
comunican un nódulo subependimario con uno o varios túberes, reflejando el tejido displásico desde su origen en progenitores ventriculares en su camino hacia el córtex (9).

\section{Nódulos subependimarios}

Característicamente abomban en la pared de los ventrículos laterales. Pueden ser nódulos individualizados o bien agruparse hasta formar conglomerados. Aunque pueden darse en cualquier lugar de la superficie de los ventrículos, son bilaterales en el 95\%, suelen agruparse en la región del surco caudotalámico, cerca del agujero de Monro $(9,11)$.

En general, los nódulos subependimarios crecen muy lentamente durante la infancia y tienen tendencia a calcificar durante los primeros meses o años de vida. En el $10-20 \%$ de los casos crecen y se transforman en astrocitomas subependimarios de células gigantes (9).

\section{Astrocitoma subependimario de} células gigantes (SEGA)

Se presenta hasta en el $10-20 \%$ de los casos. Aparece durante la infancia con un pico de incidencia en los últimos años de la infancia y la adolescencia, mientras que su aparición después de los 20 años de edad es muy poco frecuente. Suele localizarse junto a la cabeza del núcleo caudado e incluso provocan hidrocefalia por obstrucción del flujo de LCR a través del foramen de Monro en el $10 \%$ de los pacientes. El astrocitoma subependimario de células gigantes se presenta como una lesión cerebral de un diámetro mayor a $10 \mathrm{~mm}$, en la que se demuestra crecimiento entre 2 pruebas de imagen $(9,11)$.

\section{Quistes aracnoideos}

Son una manifestación reconocida recientemente y aparecen aproximadamente en un $5 \%$ de los casos, pudiendo aparecer incluso en pacientes sin túbers 0 nódulos subependimarios. Suelen ser asintomáticos. El genotipo de mayor riesgo para presentar esta manifestación es el síndrome de genes contiguos TSC2-PKD (19).

Se estima que cerca del $70-90 \%$ de los pacientes afectados por ET padecerá epilepsia, que suele ser de difícil tratamiento y es frecuentemente refractaria. En la mayoría de los casos, las crisis empezarán en el primer año de vida, en forma de espasmos infantiles (hipsarritmias de West). Las crisis más frecuentes son focales pero pueden ser de otro tipo, como generalizadas, atónicas, mioclónicas o espasmos epilépticos. Las crisis pueden aparecer a cualquier edad, incluso en la edad adulta $(5,9)$.

Por otro lado, los espasmos infantiles, las descargas epileptiformes en lóbulo temporal y el inicio precoz de las crisis también se ha relacionado con la presencia de trastorno del espectro autista.

Todo ello sugiere que un tratamiento precoz de las crisis es de gran importancia para reducir las secuelas cognitivas. Algunos autores sugieren que iniciar el tratamiento antiepiléptico al inicio de las alteraciones electroencefalográficas, antes de que aparezcan las crisis, podría reducir la severidad de la epilepsia y el riesgo de discapacidad cognitiva (9). La prevalencia de discapacidad intelectual en ET se estima entre $50-80 \%$ y 
muchos individuos con un nivel de inteligencia en rango normal pueden presentar trastornos de aprendizaje, de lenguaje y del sistema ejecutivo, siendo más frecuente el déficit de atención e hiperactividad (9).

El trastorno de espectro autista y otros trastornos psiquiátricos, como la depresión, los trastornos de comportamiento y la autoagresión son frecuentes.

La prevalencia de los trastornos de ansiedad es de $28-48 \%$ y se ha descrito un mayor riesgo de sufrir trastornos relacionados con el estrés, por ejemplo, trastorno de estrés posttraumático (9).

Los trastornos del sueño también pueden ser un problema en niños y en adultos con ET.

La disrupción del sueño se asocia con mayores problemas de comportamiento y mayor estrés parental. Un estudio polisomnográfico en niños con ET mostró varias alteraciones del sueño, principalmente una disminución de la eficiencia y del tiempo total de sueño, así como del porcentaje de sueño REM (por sus siglas en inglés, Rapid Eyes Movement) (9).

\section{MANIFESTACIONES CARDÍACAS}

El rabdomioma cardíaco se manifiesta en $50-70 \%$ de los casos, es un tumor benigno que rara vez es observado en pacientes no vinculados con el ET, usualmente no ocasionan problemas médicos severos. Su localización habitual es en los ventrículos, pueden provocar arritmias cardíacas incluyendo las arritmias atriales y ventriculares e insuficiencia cardíaca.

Aparecen durante la vida fetal por lo que suele hallarse presente ya en el nacimiento, por esto es una de las primeras manifestaciones del ET. Si se detectan un rabdomioma cardíaco de manera prenatal, ya sea por ecocardiograma o Resonancia Magnetica Nuclear (RMN), se asocia con un riesgo del $75-80 \%$ de estar afecto un $\mathrm{ET}$, el cual es aún mayor si los rabdomiomas son múltiples $(9,11,16)$.

En los lactantes afectos de ET los rabdomiomas tienden a involucionar durante los primeros meses de vida, se mantienen estables a lo largo del resto de la edad pediátrica, aunque pueden mostrar un leve crecimiento de nuevo en la adolescencia. A veces interfieren en la función valvular o provocan obstrucción de los tractos de salida. Se han descrito también arritmias, principalmente taquicardia paroxística supraventricular (síndrome de Wolff Parkinson - White (WPW)). Por ello se recomienda un seguimiento mediante electrocardiograma (ECG) periódico (cada 3-5 años) durante toda la vida, incluso después de haber demostrado la involución de los rabdomiomas $(5,9)$. También se han identificado lesiones lipomatosas cardíacas, el cual es un hallazgo frecuente en pacientes con $E T$, detectándose en aproximadamente $1 / 3$ de los pacientes a los que se les había practicado Tomografía Computarizada (TC) torácica y son asintomáticas.

\section{MANIFESTACIONES RENALES}

Las afecciones renales se observan en $50 \%$ al $80 \%$ de los pacientes, son consideradas como la segunda causa de mortalidad en el ET, usualmente se manifiestan con angiomiolipomas, quistes renales, oncocitomas y carcinomas $(9,11)$ 
1. Angiomiolipoma: es la lesión tumoral renal más frecuente. Aparece a lo largo de la infancia hasta llegar a afectar al $80 \%$ de los niños mayores 0 adultos. La complicación más frecuente de los angiomiolipomas es ET la hemorragia, que es más frecuente cuando una lesión supera los $4 \mathrm{~cm}$ de diámetro. El dolor también puede ser un síntoma importante. Cuando alcanzan gran tamaño pueden causar además problemas urológicos por desplazamiento 0 compresión de la vía urinaria. Los angiomiolipomas están compuestos de vasos, músculo liso y grasa, y no se encuentran únicamente en el riñón, sino que pueden afectar a cualquier órgano, con mayor frecuencia el hígado $(9,11,12)$.

2. Quistes: son la segunda lesión renal en orden de frecuencia. Presentes en el $17 \%$ de los pacientes en edad pediátrica y en un $47 \%$ de los adultos. A menudo son múltiples y bilaterales. Se ha descrito su involución espontánea (9).

3. Enfermedad poliquística renal: se presenta en menos del $5 \%$ de los casos. Se debe a deleciones del cromosoma 16 que afectan simultáneamente a los genes contiguos TSC2 -causante del ET- y PKD1 causante de la enfermedad poliquística renal autosómico dominante, mencionado previamente. Los afectos desarrollan una nefropatía mucho más grave y precoz que puede cursar con hipertensión arterial refractaria al tratamiento e insuficiencia renal progresiva, conduciendo al trasplante renal en las fases avanzadas de la enfermedad $(9,17)$.

\section{Angiomiolipomas}

malignos (epitelioides): la malignización de un angiomiolipoma es muy rara, pero es más frecuente en los pacientes afectos de ET que en individuos con angiomiolipomas esporádicos (9).

\section{Carcinoma de células renales} (CCR): la incidencia de CCR en los pacientes con ET es la misma que en la población general, es decir representa el $1-3 \%$ de todas las enfermedades malignas. La diferencia reside en que en los pacientes con ET el tumor se presenta a una edad media de 28 años, 25 años antes que la población general. Otro hallazgo inusual en el CCR asociado a ET es su heterogeneidad histológica. Se han descrito los subtipos de células claras, papilar, cromófobo y oncocitomas. Es un tumor de crecimiento lento y también es lenta la aparición de metástasis. EI diagnóstico diferencial entre un angiomiolipoma que crece $y$ un carcinoma de células renales es importante. Normalmente el alto contenido en grasa del angiomiolipoma permitirá distinguirlos con facilidad mediante ecografía, TC 0 , preferiblemente resonancia magnética. La punción percutánea con aguja permitirá distinguir los casos difíciles utilizando el anticuerpo monoclonal HMB-45 (4,9).

\section{LESIONES PULMONARES}

1. Linfangioleiomiomatosis (LAM): es la complicación pulmonar más 
frecuente. Como media aparece a los 33 años aunque puede presentarse ya en la adolescencia. Es mucho más frecuente en mujeres (hasta al $80 \%$ de las pacientes presenta alteración en la TC pulmonar a los 40 años), aunque puede aparecer de forma muy rara en varones. Inicialmente suele manifestarse con disnea y tos, aunque también puede producir disnea, hemoptisis, cor pulmonale, dolor torácico, neumotórax o quilotórax. Puede evolucionar hacia la insuficiencia respiratoria en la tercera o cuarta década de la vida, haciendo necesario un trasplante pulmonar en algunos casos $(4,9,14)$.

El parénquima pulmonar normal queda sustituido por incontables quistes. Histológicamente se observa proliferación de células fusiformes de músculo liso densamente empaquetadas. La LAM también puede afectar a mujeres sin ET, lo que se conoce como LAM esporádica, cuyo origen consiste en 2 mutaciones somáticas en TSC2. Un tercio de las pacientes con LAM esporádica tienen también angiomiolipomas renales. Las células de las lesiones renales presentan la misma mutación que las células de la lesión pulmonar mientras que el resto de las células del organismo no la presentan, lo que ha llevado a la hipótesis de un mecanismo de metastatización benigna como base fisiopatológica de la LAM. Es importante saber que la LAM puede exacerbarse durante la gestación, por lo que se aconseja un control muy estrecho de la función pulmonar durante el embarazo e incluso puede desaconsejarse éste, por el mayor riesgo de complicaciones como insuficiencia pulmonar, neumotórax y mayor frecuencia de parto prematuro (9).

2. Hiperplasia neumocitaria micronodular multifocal: menos frecuente que la LAM. Suele ser asintomática. Misma incidencia en hombres y en mujeres. Consiste en una proliferación de los neumocitos tipo II sin proliferación de fibras de músculo liso. Se manifiesta como densidades nodulares en las TC de pulmón (9).

3. Tumor pulmonar de células claras: al igual que la LAM, se considera una forma de PEComa (tumoración benigna de células epitelioides perivasculares) y que frecuentemente acompaña a pacientes que ya presentan LAM (9).

\section{OTRAS MANIFESTACIONES}

1. Afectación del sistema endocrino: los angiomiolipomas adrenales pueden ocurrir en una cuarta parte de los pacientes, aunque raramente producen hemorragia u otra clínica. También se han descrito hamartomas en pituitaria, páncreas, gónadas y tiroides, aunque sin causar disfunción endocrina. Se consideran un criterio menor bajo la categoría de hamartomas no renales. Los tumores pancréaticos neuroendocrinos parecen ser más frecuentes en la ET, aunque no son considerados un criterio diagnóstico por el momento (9). 


\section{Manifestaciones} gastrointestinales: son manifestaciones raras, se han descrito angiomiolipomas hepáticos en el $10-25 \%$ de los pacientes y se consideran un criterio diagnóstico mayor (como angiolipomas (2 o más) en cualquier órgano). Los pólipos rectales han sido eliminados recientemente como criterio menor de la ET por su falta de especificidad $(9,14)$.

3. Aneurismas: son manifestaciones raras, pero potencialmente mortal. Se han descrito tanto en circulación intracraneal, donde afectan principalmente a la carótida interna, y también a nivel tóraco-abdominal, afectando principalmente a aorta abdominal o arterias renales (9).

4. Lesiones óseas: se observan lesiones quísticas en falanges, matacarpo y metatarso, lesiones osteoescleróticas en columna, pelvis y cráneo, formación de nuevo hueso perióstico, osteoartropatía hipertrófica, gigantismos monomiélicos, etc.

Los quistes óseos, han sido considerados durante mucho tiempo como un criterio menor de diagnóstico hasta que en el último consenso de 2012 se decidió eliminarlos como elemento diagnóstico; debido a su inespecificidad y al hecho de que raramente se realizan radiografías de las extremidades en estos pacientes, ya que dichas lesiones suelen ser asintomáticas. A pesar de que las lesiones óseas no se consideran un criterio diagnóstico, las lesiones escleróticas óseas, frecuentemente detectadas en raquis al realizar TC pulmonar 0 RMN abdominal, son muy frecuentes en ET y suelen ser asintomáticas. Es importante conocerlas ya que pueden confundirse con metástasis osteoblásticas. También se ha descrito de forma rara la afectación ósea en forma de displasia fibrosa $(9,18)$.

5. Cordoma: es un tumor raro que parece ser ligeramente más frecuente en ET. Los cordomas asociados a ET presentan una serie de peculiaridades respecto a los cordomas esporádicos, como aparecer mucho más precozmente, afectar con mayor frecuencia al sacro y un mayor índice de supervivencia tras el tratamiento (9).

\section{DIAGNÓSTICO}

Actualmente, existen varios criterios diagnósticos para $\mathrm{ET}$, los que rigen actualmente son del Grupo Internacional de Esclerosis Tuberosa actualizado en el 2012, con estos criterios se puede clasificar de la siguiente manera. Ver TABLA 1:

- diagnóstico definitivo: dos criterios mayores o uno mayor con $\geq 2$ menores.

- diagnostico posible con un criterio mayor o $\geq 2$ menores.

Para poder definir o establecer algunos de estos criterios se ameritan estudios específicos que bien dependerán de la edad del individuo; en la TABLA 2, se demuestra algunas evaluaciones Recomendadas para el estudio de paciente con sospecha de ET. La consejería genética en los casos de herederos por ET se hace explicando 
TABLA 1. Criterios diagnósticos para la esclerosis tuberosa.

\section{A) GENÉTICOS}

La identificación de una mutación patogénica en TSC1 o TSC2 para realizar el diagnóstico definitivo.

Del 10 al 25\% de los pacientes no tienen mutación identificada en los genes TSC1 o TSC2 y esto no excluye el diagnóstico, por lo que se deben emplear los criterios clínicos.

\section{B) CLínICOS}

\section{CRITERIOS MAYORES}

1. Angiofibromas faciales $(\geq 3)$ o placas en la frente

2. Máculas no traumáticas hipomelánicas ( $\geq 3$ al menos de $5 \mathrm{~mm}$ de diámetro)

3. Fibromas ungueales o periungueales no traumaticos $(\geq 2)$

4. Parche lijoso o placas de Chagrín (nevus de tejido conectivo)

5. Múltiples hamartomas nodulares retinianos

6. Tuberomas o displasias corticales (incluye líneas de migración radial de sustancia blanca cerebral)

7. Nódulos subependimarios

8. Astrocitoma subependimario de células gigantes

9. Rabdomioma cardíaco, único o múltiples

10. Linfangiomatosis pulmonar *

11. Angiomiolipoma $(\geq 2)$ *

\section{CRITERIOS MENORES}

1. Pozos o Pits distribuidos al azar en el esmalte dental $(>3)$

2. Fibromas orales $(\geq 2)$

3. Hamartoma no renal (confirmación histológica)

4. Placa acrómica retiniana

5. Lesiones cutáneas en confeti

6. Quistes renales múltiples (confirmación histológica)

*Una combinación de estos dos criterios mayores sin otros hallazgos no cumplen con el criterio de diagnóstico definitivo. Fuente. Hallazgos clínicos y epidemiológicos en la neurofibromatosis tipo 1 y el complejo esclerosis tuberosa en una serie de pacientes pediátricos (2).

TABLA 2. Evaluaciones recomendadas para el estudio de pacientes con sospecha de esclerosis tuberosa

- Ecocardiografía en niños menores de 5 años como parte del diagnóstico.

- Electrocardiograma como una investigación de referencia para excluir defectos de la conducción cardíaca y arritmias.

- Ecografía renal en el momento del diagnóstico para la detección de enfermedad renal poliquística asociada con deleciones de genes contiguos de los genes TSC2 y PKD2.

- Seguimiento ecográfico renal a los 5, 8, 12 y 16 años, según corresponda.

- Electroencefalograma para la evaluación de las convulsiones.

- RMN craneal a la edad de 2 años o más, si aún no se ha realizado como parte del estudio de diagnóstico

- Genotipo

Fuente. The Tuberous Sclerosis 2000 Study: presentation, initial assessments and implications for diagnosis and management (13)

que tienen un $50 \%$ de probabilidad de tener hijos con la enfermedad y que no es posible predecir cual será el grado de expresión del fenotipo, este puede ser desde leve con solo hallazgos en piel hasta severo con discapacidad intelectual, convulsiones de difícil manejo, tumores en sistema nervioso central, corazón, riñones o hígado (1). El diagnóstico molecular tiene 
limitaciones ocasionadas por el tamaño de los genes, la heterogeneidad mutacional y el fenómeno de mosaicismo somático. Existen diferentes técnicas para la realización de estas pruebas, una de ellas es la secuenciación del gen TSC2 permite detectar mutaciones en el $60-70 \%$ de los casos esporádicos y en el $50 \%$ de los familiares, mientras que en el gen TSC1 se detectan mutaciones en el $15 \%$ y $30 \%$, de los casos esporádicos y familiares (8).

La secuenciación no permite detectar deleciones grandes 0 rearreglos, eventos que son más frecuentes en el gen TSC2. Siempre que se realiza esta técnica, existe la posibilidad de encontrar cambios que no representan un daño en la función de los productos protéicos, variaciones que tienen un significado clínico desconocido y demandan la realización de estudios en los padres 0 en familiares afectados (8).

Técnicas como el MLP (Multi-Layer Protocol), el FISH (Fluorescence in situ hybridization), o el Southern Blot se emplean cuando el análisis por secuenciación es negativo, lo que permite identificar mutaciones en el $0,5 \%$ al $5 \%$ de los casos. Por el fenómeno de mosaicismo, ante la ausencia de resultados positivos en los estudios moleculares, es recomendable realizar los análisis en tejidos diferentes a la sangre, incluyendo los tumores, la saliva, la piel y los folículos pilosos (8).

Los padres de un paciente con diagnóstico de ET, deben ser evaluados clínicamente en busca de signos que sugieran el diagnóstico. La búsqueda de máculas hipocrómicas debe incluir en lo posible, la lámpara de Wood. Si uno de los padres está afectado, el riesgo de recurrencia del
ET en la descendencia es del $50 \%$, sin embargo, si no hay evidencia clínica de enfermedad en los progenitores, no es posible descartar el fenómeno de mosaicismo, motivo por el que es importante realizar los análisis moleculares en sangre; en ausencia de mutaciones, en los elementos formes de la sangre, el riesgo de recurrencia es del $1 \%$ a $2 \%(8,18)$.

\section{TRATAMIENTO}

\section{- TRATAMIENTO CON INHIBIDORES DE LA VÍA MTOR}

Puesto que las células patológicas en los pacientes con ET presentan una activación patológica de la vía mTOR, los inhibidores de mTOR, como sirolimus (rapamicina) y everolimus, tiene un efecto poderoso como antiproliferativo e inmunosupresor, por lo que se están utilizando como tratamiento de algunas anomalías asociadas a ET (9).

La utilidad de estos fármacos modificadores ya está reconocida para el tratamiento del astrocitoma subependimario de células gigantes y de los angiomiolipomas renales. También parece que pueden ser útiles en el manejo de otras manifestaciones de la enfermedad, ya que se ha visto en modelo de ratones que mejoran la mielinización, la arquitectura citopatológica y reestablece la función sináptica, por lo que se cree que ayuda a prevenir la epilepsia, el trastorno del espectro autista, déficit en el aprendizaje, epilepsia, la linfangioleiomiomatosis, los angiofibromas, o los rabdomiomas cardíacos sintomáticos, aunque se precisan más estudios para generalizar su uso clínico en estas indicaciones $(4,9)$.

En estudios realizados con rapamicina, 
se ha observado que tiene actividad sobre los receptores intracelulares FKBP12, disminuye la producción del VEGF, lo que es benéfico para tumores vasculares por sus efectos antiangiogénicos. Rauktys, demostró que la administración tópica de rapamicina es efectiva para tumores relacionados con esclerosis tuberosa en modelos animales, lo cual pudiera ser efectivo en manejo de angiofibromas faciales (19).

En caso de recibir tratamiento con inhibidores de mTOR, dado que producen inmunosupresión, y por tanto, más riesgo de infecciones, es necesario seguir un correcto calendario vacunal, el cual incluye vacunación anual contra la gripe. En el caso de que se deba administrar una vacuna de virus atenuados, es aconsejable la interrupción del tratamiento con un inhibidor de mTOR 2 semanas antes de realizar la vacunación, que podrá reanudarse 2 semanas después (9).

\section{- TRATAMIENTOS ESPECÍFICOS SEGÚN LA ESPECIALIDAD}

\section{NEUROLOGÍA Y PSIQUIATRÍA}

\section{Astrocitoma subependimario de} células gigantes: la resección quirúrgica está indicada para tumores sintomáticos de forma aguda y una derivación ventriculoperitoneal puede ser necesaria en estos casos. En caso de tumor asintomático en crecimiento, tanto la resección quirúrgica como el uso de inhibidores de mTOR son adecuadas y la decisión de la opción más adecuada se realizará en cada caso individualmente (9).
2. Tratamiento de la epilepsia: el mejor tratamiento inicial en el paciente es la terapia con medicamentos anticonvulsivantes y dieta cetogénica. Se ha observado que la vigabatrina, es un inhibidor de la transaminasa del ácido gaminobutirico, es el anticonvulsivante de elección en estos pacientes con ET. Si el tratamiento anticonvulsivante y la dieta cetogénica no son efectivas, la intervención neuroquirúrgica se puede considerar, realizando un EEG para localizar el foco epileptógeno para localizar la lesión cortical. La resección del foco epileptogeno en estos pacientes, elimina las crisis convulsivas en un 25 al $69 \%(17,19)$. En el estudio realizado en el Hospital Nacional de Niños (HNN) en Costa Rica, dentro de las combinaciones que lograron una mayor reducción de las crisis en un mayor número de pacientes, se encontraron con frecuencia la vigabatrina y la carbamacepina.

La carbamacepina es un fármaco muy útil en crisis de inicio parcial, como suelen ser las crisis en la ET, lo que justificaría este hallazgo (3).

En varios estudios han demostrado que la hormona adrenocorticotropa y los esteroides son tratamientos efectivos en los espasmos infantiles, y se pueden utilizar como terapias complementarias (3). Con relación a los fármacos inhibidores de la vía mTOR se mantiene a la espera de que haya mayor evidencia que permita incluirlos como tratamiento habitual de la epilepsia en ET, sin embargo, se podría tomar en consideración en casos de epilepsias refractarias. Varios experimentos europeos en 
TABLA 3. Recomendaciones para el manejo clínico de la epilepsia de ET, Consenso Europeo

\section{Tratamiento de los espasmos infantiles}

1a línea: la vigabatrina sigue siendo la primera línea de tratamiento en espasmos infantiles, a la minima

- dosis efectiva y durante el menor tiempo posible para minimizar el riesgo de toxicidad retiniana. También puede ser de elección en el caso de crisis focales en el primer año de vida.

- $\quad 2^{a}$ línea: corticosteroides o ACTH en caso de hipsarritmia. Si no hay hipsarritmia pero se observan alteraciones focales/multifocales puede utilizarse topiramato.

- 3a línea:

1. Dieta cetogénica, otros fármacos antiepilépticos.

2. Cirugía: debe ser considerada de forma temprana ante una epilepsia fármaco-resistente ya que un foco de displasia puede manifestarse en forma de espasmos infantiles

\section{Tratamiento de las crisis focales}

- $\quad 1^{a}$ línea: vigabatrina en menores de un año y otros fármacos que potencien la acción inhibitoria del GABA (por ejemplo: carbamacepina, topiramato) después del año de vida.

- $\quad 2^{a}$ línea: cirugía (el éxito depende de la precisa localización del foco epiléptico).

- $\quad 3^{a}$ línea:

1. Dieta cetógena.

2. Estimulación del nervio vago: muy raramente consigue dejar libre de crisis pero se ha descrito una disminución significativa en el número de crisis.

Cirugía: está indicada de forma precoz en epilepsia refractaria, si de identifica claramente la lesión causante de las crisis. Dado que lesiones focales pueden dar lugar a crisis bilaterales y espasmos infantiles, puede considerarse cirugía en estos casos. La cirugía está contraindicada en caso de múltiples tipos de crisis, pero debe ser considerada si hay un tipo predominante de crisis que altera de forma importante la calidad de vida.

Fuente. Complejo esclerosis tuberosa (9).

ET publicaron en consenso unas recomendaciones para el manejo clínico de la epilepsia en ET, ver TABLA 3.

\section{Tratamiento} de las manifestaciones psiquiátricas: deberán tratarse según indicación clínica. Los inhibidores selectivos de la recaptación de serotonina han mostrado su utilidad como antidepresivos y en los trastornos por ansiedad, incluyendo el estrés post-traumático, que pueden presentar estos pacientes (9).

\section{CARDIOLOGÍA}

Los rabdomiomas cardíacos suelen ser asintomáticos, pero si son grandes $u$ obstruyen un orificio cardíaco pueden ocasionar insuficiencia cardíaca. Dado que la tendencia natural es hacia la involución, la insuficiencia cardíaca es más frecuente en los recién nacidos y lactantes de corta edad. Se ha descrito crecimiento de rabdomiomas cardíacos al iniciar tratamiento con ACTH en lactantes con espasmos infantiles. Las arritmias cardíacas son menos frecuentes, pero deben buscarse mediante ECG. Se ha descrito la aparición de bloqueo auriculoventricular al iniciar tratamiento médico de la epilepsia con carbamazepina, por lo que es recomendable un control ECG estricto en caso de uso de este fármaco (9).

\section{NEFROLOGÍA}

Las lesiones renales benignas como quistes o angiomiolipomas no requieren ningún tratamiento. En caso de duda en la distinción entre lesión benigna o maligna o bien en caso de crecimiento importante que implique riesgo de complicaciones puede estar indicada la 
extirpación de la lesión. La embolización arterial percutánea seguida de corticoides es la técnica de elección para el angiomiolipoma que se presenta como sangrado (9).

En caso de angiomiolipomas asintomáticos en crecimiento $(>3 \mathrm{~cm})$, la terapia de $1^{\text {a }}$ línea es la administración de un inhibidor de mTOR.

Como terapia de segunda línea se consideran otras técnicas como la embolización selectiva o la resección con máxima conservación de parénquima renal, que son terapias aceptables en caso de angiomiolipoma asintomático en crecimiento para evitar el riesgo de sangrado. La asociación de enfermedad poliquística renal puede provocar hipertensión arterial e insuficiencia renal que pueden llevar a la necesidad de diálisis o incluso de trasplante renal. No se ha observado la reaparición de las lesiones en el riñón trasplantado (9).

\section{NEUMOLOGÍA}

Los inhibidores de mTOR pueden usarse para el tratamiento de pacientes con linfangioleiomiomatosis moderadasevera 0 rápida progresión. La enfermedad grave puede requerir trasplante pulmonar (9).

\section{CUTÁNEAS Y ORALES}

Los angiofibromas faciales pueden extirparse o mejorar notablemente con tratamiento con láser. El láser de argón o los pulsed dye lasers son preferibles para las lesiones muy vascularizadas, las fibróticas se tratan preferentemente con láser de CO2. También pueden tratarse con inhibidores de mTOR tópicos. Los fibromas orales pueden requerir resección. Debido a que un estudio muy reciente demuestra que un gran número de mutaciones somáticas en los angiofibromas faciales son inducidas por los rayos ultravioleta, es recomendable limitar la exposición a la radiación solar

\section{REFERENCIAS}

1. Arango J, Delgado J, Saldarriaga W. Esclerosis tuberosa, diagnóstico fetal y materno. Revista chilena de obstetricia y ginecología. 2015 Dec;80(6):475-480. https://doi.org/10.4067/s071775262015000600007

2. Cammarata-Scalisi F, Stock F, Velazco N, Da Silva G, Lacruz-Rengel MA, Avendaño A. Hallazgos clínicos y epidemiológicos en la neurofibromatosis tipo 1 y el complejo esclerosis tuberosa en una serie de pacientes pediátricos. Boletín Médico del Hospital Infantil de México. 201901 29;75(5).https://doi.org/10.24875/bmhim.m18000035

3. Ulate-Campos A, Benavides-Lara A, Hernández L. Caracterización de la población pediátrica costarricense con esclerosis tuberosa y descripción del comportamiento de la epilepsia asociada. . Revista de neurología. 2013 Diciembre 1;57(11):489-494. Disponible en:http://inciensa.sa.cr/actualidad/hisotico noticias/Caracterizacion poblacion pediatrica con escler osis tuberosa.aspx

4. Cammarata-Scalisi, F, Lacruz-Rengel, MA, Stock, F, Vidales, C, Callea, M. ASPECTOS CLÍNICOS Y GENÉTICOS DEL COMPLEJO ESCLEROSIS TUBEROSA. Archivos Venezolanos de Puericultura y Pediatría [Internet]. 2017;80(1):27-33. Recuperado de: https://www.redalyc.org/articulo.oa?id=367951839007 
5. Sánchez-Meza E, Ancer-Arellano J, Villarreal-Villarreal CD, Cárdenas-de la Garza JA y col. Características clínicas y epidemiológicas de esclerosis tuberosa en un centro de referencia dermatológico del noreste de México. Dermatol Rev Mex. 2018 noviembre -diciembre;62(6):475-485. Disponible en:http://www.medigraphic.com/pdfs/derrevmex/rmd-2018/rmd186d.pdf

6. Crino PB, Nathanson KL, Henske EP. The Tuberous Sclerosis Complex. New England Journal of Medicine. 200609 28;355(13):1345-1356. https://doi.org/10.1056/nejmra055323

7. Maita F, Mamani C, Morales L, Arnés R, Sánchez K, Galvarro S. Complejo esclerosis tuberosa: diagnóstico, manejo pre y posnatal: reporte de un caso clínico. Gac Med Bol. 2017 Enero junio;40(1):41-45. Disponible en: http://www.scielo.org.bo/pdf/gmb/v40n1/v40n1a9.pdf

8. Medina-Malo C, Carreño O, Vélez A, Lizcano LA, Darío Ortiz L, Becerra H, Cardona AF. Complejo esclerosis tuberosa. Acta Neurol Colomb. 2012 Marzo;28(1):11-23. Disponible en:http://www.actasdermo.org/es-esclerosis-tuberosa-enfermedad-pringle-bourneville-articulo$\underline{13026854}$

9. Boronat S, Sábado C, Vendrell T. Complejo esclerosis tuberosa. Grupo de trabajo sobre cáncer en síndromes genéticos polimalformativos. 2015 Julio. 1-34. Disponible en:https://www.orpha.net/data/patho/Cpg/es/ComplejoEsclerosisTuberosa ESes CPG ORPHA805.pdf

10. Escariz L, Mederos K, Chávez V, Segers D, Andrade M. Esclerosis Tuberosa: a propósito de un caso. Revista San Gregorio. $2018 \quad$ Noviembre;1(24):44-49. Disponible en:http://revista.sangregorio.edu.ec/index.php/REVISTASANGREGORIO/article/view/724

11. Núñez E, Bonilla Y, Varela D. Actualización de métodos diagnósticos en el Complejo de Esclerosis Tuberosa. Revista Mexicana de Neurociencia. 2016 Julio - agosto;17(4):86-95. Disponible en:http://revmexneuroci.com/wp-content/uploads/2016/10/RevMexNeu-No-4-Jul-Ago-2016-86-95$\underline{\text { R.pdf }}$

12. Monteiro T, Garrido C, Pina S, Chorão R, Carrilho I, Figueiroa S, Santos M, Temudo T. Esclerosis tuberosa: caracterización clínica e intento de correlación fenotipo/genotipo. Anales de Pediatría. 2014 Nov;81(5):289-296. https://doi.org/10.1016/j.anpedi.2014.03.022

13. Weisenfeld NI, Peters JM, Tsai PT, Prabhu SP, Dies KA, Sahin M, Warfield SK. A Magnetic Resonance Imaging Study of Cerebellar Volume in Tuberous Sclerosis Complex. Pediatric Neurology. 2013 02;48(2):105-110. https://doi.org/10.1016/j.pediatrneurol.2012.10.011

14. Rubilar C, Lopez F, Troncoso M, Barrios A, Herrera L. Estudio clínico genético en pacientes con complejo de esclerosis tuberosa. Rev Chil Ped. 2017;88(01): 41-49. Disponible en:http://www.revistachilenadepediatria.cl/index.php/rchped/article/view/213

15. Vanegas C, Bustos C. Complejo de esclerosis tuberosa de diagnóstico tardío. Revista Argentina de Radiología / Argentinian Journal of Radiology. 201803 23;82(03):131-133. https://doi.org/10.1055/s$\underline{0038-1639380}$

16. Fernández Concepción Otman, Gómez García Ariel, Sardiñaz Hernández Norberto. Esclerosis Tuberosa. Revisión. Rev Cubana Pediatr [Internet]. 1999 Sep; 71( 3 ): 160-167. Disponible en:http://scielo.sld.cu/scielo.php?script=sci arttext\&pid=S0034-75311999000300006\&lng=es

17. Osborne JP, Merrifield J, O'Callaghan FJK. Tuberous sclerosis--what's new?. Archives of Disease in Childhood. 200809 01;93(9):728-731. https://doi.org/10.1136/adc.2006.094938

18. Ruiz Villaverde R, Blasco Melguizo J, Cruz Martín Sánchez M, Naranjo Sintes R. Esclerosis tuberosa. Enfermedad de Pringle Bourneville. Actas Dermo-Sifiliográficas. 2002 Oct;93(1-2):17.https://doi.org/10.1016/s0001-7310(02)79162-x 